\title{
Actividades de servicio y servitización: oportunidades para una economía circular más sostenible
}

Service Activities and Servitisation: Opportunities for a More Sustainable Circular Economy

Ángeles Pereira Sánchez

Grupo Icede, Departamento de Economía Aplicada, Universidad de Santiago de Compostela (USC) angeles.pereira@usc.es 
Los urgentes retos medioambientales que la sociedad debe afrontar en el futuro próximo reclaman la reconsideración del modelo de producción y consumo lineal dominante, así como el paso a una economía más circular y sostenible. EI objetivo de este artículo es realizar un análisis teórico-conceptual sobre el papel de las actividades de servicios, junto con el de los servicios relacionados con la producción y los productos, en la desmaterialización y ecologización del sistema socioeconómico. Para ello, se realizó una revisión bibliográfica-documental centrada en tres dimensiones: en primer lugar, la naturaleza de los servicios y las características que permiten el desarrollo de una economía desmaterializada; en segundo lugar, la economía de servicios funcional en el marco de una economía circular; $y$, en tercer lugar, la servitización como un modelo de negocio innovador, basado en la hibridación de productos y servicios, con potencial para lograr beneficios ambientales. A partir del análisis desarrollado, en la sección de conclusiones se presenta una propuesta clasificatoria sugerida de las diferentes actividades de servicio con capacidad para apoyar la transición hacia una economía a lo largo del ciclo de vida de los productos. Se distingue así entre diferentes actividades de servicio y de servitización en la fase de producción, en la fase de uso/consumo y en la fase de recuperación al final de ciclo.

Palabras clave: servitización, servicios, modelos de negocio, economía circular, sostenibilidad

The urgent environmental challenges that society must face in the near future call for a reconsideration of the dominant linear production and consumption model, and a shift to a more circular and sustainable economy. The aim of this paper is to carry out a theoretical-conceptual analysis of the role of service activities, as well the one of services related to production and products, in the dematerialization and greening of the socio-economic system. To this end, a literature review was conducted focusing on three dimensions: firstly, the nature of services and the characteristics that enable the development of a dematerialized economy; secondly, the functional service economy in the framework of a circular economy; and, thirdly, servitization as an innovative business model, based on the hybridization of products and services, with the potential to achieve environmental benefits. Based on the analysis developed, the conclusions section presents a suggested classification of the different service activities with the capacity to support the transition to a more sustainable economy throughout the life cycle of products. A distinction is thus made between different service and servitization activities in the production phase, in the use/consumption phase and in the end-of-cycle recovery phase.

Keywords: servitization, services, business models, circular economy, sustainability 


\section{Introducción}

El cambio estructural que caracteriza a la economía mundial en las últimas décadas viene determinado por el crecimiento de las actividades de servicios, el desarrollo de las tecnologías de la información y de nuevas actividades, y por la creciente imbricación entre las industrias manufactureras y las de servicios, entre otros factores (Rubalcaba Bermejo, 2008). Los servicios se han presentado en las industrias manufactureras durante mucho tiempo (por ejemplo, el transporte, la comercialización, etc.), pero la tendencia más reciente es hacia la fusión de servicios y productos en la propuesta de valor de las actividades manufactureras tradicionales.

A lo largo de las dos últimas décadas, la innovación en los servicios se ha estudiado desde el punto de vista económico (Gallouj \& Savona, 2008; Toivonen \& Tuominen, 2009; Toivonen, 2010) como vía para crear beneficios para la organización que la desarrolla y la pone en práctica. Asimismo, la contribución de los servicios a la innovación de otras empresas también ha sido ampliamente estudiada (Bessant \& Bush, 2000; Boden \& Miles, 2000; Camacho \& Rodríguez, 2009; Vence \& González-López, 2009). En cambio, pocos estudios han puesto el foco en la ecoinnovación de servicios o en la innovación de los servicios para el logro de una mejora medioambiental (Cainelli \& Mazzanti, 2013). Este artículo se centra en el análisis de este último aspecto y, en concreto, en el papel de los servicios en una economía circular (EC). Esta se define por oposición a la economía lineal (extraer, usar, tirar) como un nuevo paradigma de producción y consumo centrado en consumir menos recursos materiales y prolongar la vida útil de los productos y materiales, minimizando al mismo tiempo la generación de residuos.

A falta de consenso académico para una definición de EC (Reike et al., 2018; Kirchherr et al., 2017; Korhonen et al., 2018), en este artículo partimos principalmente de dos propuestas, seleccionadas por su ya largo recorrido y por poner de relieve dos aspectos centrales en nuestro análisis: la relación entre la economía industrial y los servicios, y el potencial de los servicios para cumplir con los principios de la EC. En primer lugar, la Fundación Ellen MacArthur (EMF, por sus siglas en inglés), pionera en la promoción de este nuevo modelo, define la EC como "una economía industrial que sea restauradora por intención; que aspire a depender de las energías renovables; que minimice, rastree y elimine el uso de productos químicos tóxicos; y que erradique los residuos mediante un diseño cuidadoso» (EMF, 2013, p. 22). Por otra parte, Walter Stahel (2010, 2013), conocido referente de la "economía del desempeño», destaca el profundo cambio económico de una economía lineal a una EC. Esta última se centra en la optimización de stocks y, por tanto, sustituye el valor añadido por la conservación de valor y el valor de uso por el valor residual.

En este artículo analizamos la contribución de los servicios y la servitización (hibridación de productos y servicios) a la realización de los principios de la EC. Desde nuestro punto de vista, la EC establece una nueva orientación-guía para las actividades económicas, entre las cuales los servicios están llamados a cumplir un papel principal. El artículo está estructurado de la siguiente forma: en el siguiente apartado, presentamos brevemente la metodología seguida para la realización de la revisión bibliográfica. A continuación se presentan los resultados, estructurados en tres epígrafes de acuerdo con los siguientes propósitos: 1) identificar la naturaleza y las características de las actividades 
de servicios que son favorables a la mejora medioambiental; 2) analizar las aportaciones de los servicios a la sostenibilidad en el marco de la EC y de la economía de servicios funcional; y 3) analizar la estrategia concreta de la servitización desde un punto de vista teórico y estudiar la evidencia empírica sobre su contribución al mejor desempeño ambiental. Cerramos el artículo con un apartado de conclusiones, incluyendo una propuesta sobre las oportunidades de los servicios para la EC.

\section{Metodología}

Este artículo se elabora con base en una revisión bibliográfica-documental del tema de investigación, realizada con el objeto de dar respuesta a la pregunta "¿cuál es el papel de las actividades de servicio, y de los servicios ligados a la producción y al producto, en una economía circular más sostenible?». Para ello, la selección de la literatura pertinente se ha basado en la búsqueda a través de la combinación de dos bases de datos: Scopus y Google Académico. Las palabras clave utilizadas han sido "servicios y medio ambiente», "servicios y sostenibilidad", "servicios y economía circular», "servitización (o servicizing) y sostenibilidad», y "servitización (o servicizing) y economía circular», generando resultados de entre dos y más de ochenta referencias. A través de ambas fuentes documentales hemos tratado de identificar las referencias que representan un claro aporte al conocimiento para nuestro objeto de estudio. El primer criterio de selección de fuentes se basó en identificar los principales aportes teóricos al objeto de investigación. El segundo, aplicado a la literatura sobre "servitización» —que, por cierto, generó un mayor número de referencias—, se basó en las revisiones sistemáticas de esta literatura, así como en la selección de estudios de caso que permitiesen constatar desde una perspectiva más empírica el papel desarrollado por los servicios en la EC.

\section{Los servicios y los beneficios para el medio ambiente}

En el pensamiento económico clásico, las actividades de servicios se definieron como actividades improductivas e inmateriales (Cuadrado Roura \& Rubalcaba Bermejo, 2000). Esas ideas iniciales llevaron al supuesto de que las economías terciarizadas serían cada vez más desmaterializadas y, por tanto, tendrían un impacto medioambiental menor. Debido al resultado inmaterial, evanescente y transitorio de los servicios, se asume que no producen daños en el entorno natural, contrariamente a lo que sucede con la producción industrial, que transforma las materias primas en bienes físicos y daña el medio ambiente tanto en la producción como mediante el consumo (Djellal \& Gallouj, 2016). La supuesta inmaterialidad de las economías terciarizadas no encuentra, sin embargo, consenso académico. De acuerdo con Gadrey (2010), los únicos indicadores verosímiles del materialismo creciente o decreciente de las sociedades son el volumen total de apropiación de recursos (finitos) y de residuos. Y estos se han incrementado de manera continuada lo largo del siglo XX y de forma acelerada en las décadas más recientes (Krausmann et al. 2018), superando la biocapacidad de la Tierra.

Para diferenciarlas de otras actividades (agrarias, industriales, etc.), Boden y Miles (2000) definen los servicios como el conjunto de actividades que efectúan cambios en 
el estado de 1) el entorno natural (distintos de los relativos a la extracción de materias primas), como la gestión de residuos, los procesos de descontaminación o la conservación de parques; 2) artefactos producidos por el sector industrial, como la instalación, reparación y mantenimiento, los bienes de transporte, servicios de edificación, o el comercio al por mayor y menor; 3) las personas, como servicios sanitarios y educativos, de hostelería y consumo, peluquería, transporte público; y 4) los símbolos (es decir, información), como los servicios de conocimiento (que aportan inteligencia para mantener cualquiera de las otras actividades mencionadas), los servicios de entretenimiento, y los servicios de comunicación y telecomunicaciones.

Aunque los servicios como resultado implican una realidad inmaterial, su creación y prestación se basa en numerosas fuentes de materialidad (Djellal \& Gallouj, 2016). Primero, los servicios se definen por su médium o target, que es la realidad modificada y puede presentar un grado variable de materialidad (un bien físico, información codificada, individuos, organizaciones, etc.). Segundo, los servicios son producidos y prestados en espacios materiales (back office, donde se realiza la transformación material o informacional del target; y front office, área de relación con el cliente). Tercero, los factores de producción empleados en la producción del servicio suponen en muchos casos una elevada intensidad material (por ejemplo, servicios de maquinaria agrícola, redes e infraestructuras de prestación de servicios).

Cabe precisar que reconocer las diferentes fuentes de materialidad de los servicios no implica el rechazo directo de los beneficios ambientales asociados a estos. De hecho, los mismos autores citados anteriormente sugieren que la sostenibilidad y la ecologización son tendencias que definirán en el futuro la naturaleza de los servicios. Gadrey (2010) relaciona el desarrollo de los servicios con la relocalización de otras actividades productivas, de modo que augura el aumento del empleo en los servicios, en parte motivado por el giro hacia la sostenibilidad y hacia un modelo no productivista de las actividades primarias e industriales. Además, considera que los servicios experimentarán ellos mismos numerosas innovaciones con el objetivo de reducir su huella medioambiental y, a la vez, desarrollar servicios locales que sean beneficiosos desde el punto de vista ambiental y social. Igualmente, la importancia relativa de los servicios aumentará por el declive de las formas de producción que devoran energía y recursos ecológicos por unidad de servicio final y producen elevados volúmenes de emisiones, incluidos los gases de efecto invernadero.

Las vías de desmaterialización y ecologización de los servicios pueden ser variadas (Djellal \& Gallouj, 2016; Gallouj, 2000). En primer lugar está la desmaterialización del médium o target del servicio, que correspondería a nivel macro con la creciente relevancia de los servicios de carácter informacional, cognitivo y relacional frente a los servicios materiales. A nivel micro, aumentarían las operaciones de procesamiento de medios inmateriales en las organizaciones respecto a las operaciones de medios materiales. En este sentido, las trayectorias tecnológicas y de innovación propias de cada actividad de servicio pueden condicionar la relativa desmaterialización del target. Concretamente, la desmaterialización del servicio se asocia con el aumento de las trayectorias de innovación inmaterial (servicio puro), cognitivo (metodológico) e informativo (software).

En segundo lugar, tenemos la desmaterialización y ecologización de los espacios de producción/consumo; y, en tercer lugar, la desmaterialización y ecologización de los factores 
de producción, condicionada también por las posibles evoluciones de las trayectorias de innovación de los servicios. Específicamente, los autores distinguen: 1) el aumento de las trayectorias de innovación inmaterial (como métodos de consultoría, protocolos de salud y limpieza) y de los servicios puros; 2) el fortalecimiento de la dimensión inmaterial en una determinada estrategia de innovación (como el desarrollo y la adopción de tecnologías más limpias y eficientes en el consumo energético); 3) la hibridación de diferentes trayectorias, por ejemplo, el uso de las tecnologías de la información y la comunicación (TIC), que permite ahorrar energía (redes inteligentes) o desplazamientos (cirugía en remoto); y 4) los cambios en los regímenes de uso y propiedad de los bienes materiales.

En síntesis, los servicios pueden contribuir a un mejor desempeño medioambiental de las economías al menos de dos modos: por un lado, pueden diseñarse a sí mismos para ser más ecoeficientes y respetuosos con el medio ambiente; y, por otro, pueden contribuir a disminuir el impacto medioambiental de otras actividades.

\section{La EC y la economía de servicios funcional}

En los últimos años, la EC ha emergido como un nuevo paradigma de producción y consumo, alineado con el desarrollo sostenible, que desvincula la extracción de recursos materiales y el consumo de energía del crecimiento, al tiempo que promueve un uso ecoeficiente de los recursos, basado en la recuperación y el reciclaje total de los materiales usados y la transformación de los residuos en nuevos recursos (EMF, 2013; Ghisellini et al., 2014). La realización y consecución de la EC requiere la adopción de una serie de principios guía por parte de los diferentes agentes sociales. En síntesis: 1) conservar y aumentar el capital natural, controlando los stocks finitos y equilibrando los flujos de recursos renovables; 2) optimizar el rendimiento de los recursos, ciclando de manera continua los productos, componentes y materiales; y 3) promover la eficacia del sistema mediante un diseño que evite las externalidades negativas (EMF, 2013).

Desde el punto de vista empresarial, Bocken et al. (2016) identifican diferentes estrategias prácticas para aplicar la circularidad. Para ralentizar la circulación de recursos a lo largo del ciclo de vida, las estrategias clave son: 1) satisfacer las necesidades sin transferir la propiedad del producto mediante modelos de acceso y rendimiento; 2) extender el valor del productos mediante actividades de refabricación y reacondicionamiento; 3) el modelo clásico de larga vida mediante el diseño de productos sostenibles (durabilidad y reparación); y 4) el fomento de la suficiencia a través de la ampliación de la vida útil del producto hasta el nivel del usuario final mediante la durabilidad, mejora, reparación y las garantías.

Además, para cerrar los ciclos entre el postuso y la producción, se sugieren dos estrategias: 1) ampliar el valor del recurso, convirtiendo los residuos en un recurso valioso; y 2) establecer modelos basados en la simbiosis industrial, de modo que los subproductos de un proceso industrial se conviertan en insumos para otra industria.

A estas estrategias hay que añadir la reducción, centrada en la utilización de menos productos, componentes, materiales y energía durante las fases del ciclo de vida del producto (desde el diseño hasta la recuperación); la regeneración, que se refiere a la utilización de materiales renovables y no tóxicos, así como de energía renovable; y la 
información, que se basa en el uso de la tecnología de la información para apoyar la EC (Konietzko et al., 2020).

De esta manera, la EC asienta una serie de principios guía y orientaciones prácticas que pueden abrir nuevas oportunidades para los servicios. En este sentido, cabe destacar a la economía de servicios funcional como complementaria a la EC. En el libro The Performance Economy, Stahel (2010) argumenta que la "economía del desempeño» o la economía de servicios funcional es competitiva y sostenible porque

optimiza la utilización (o función) de los bienes y servicios y, así, la gestión de riqueza existente (bienes, conocimiento y naturaleza). El objetivo económico de la economía funcional es crear el mayor valor de uso posible durante el mayor tiempo posible consumiendo el menor número posible de recursos materiales y energía (Stahel, 1997).

Así, concibe esta economía guiada por una propuesta integral basada en producir, vender y administrar el desempeño de los productos a lo largo del tiempo. De esta forma, los servicios se caracterizarían por satisfacer las necesidades de los consumidores en base a la funcionalidad de los productos o el resultado de las actividades de servicio.

Las estrategias para producir funcionalidad se construyen sobre la creación de riqueza a partir del conocimiento, el aprovechamiento del progreso científico y tecnológico, la prevención del riesgo y la búsqueda de la suficiencia (reducción de las fases de la vida del producto, utilizando menos recursos y generando menos residuos y emisiones), así como de las soluciones sistémicas inteligentes (basadas en la simbiosis, los bucles virtuosos, etc.). La venta de funcionalidad puede realizarse a través de varias estrategias (Stahel, 2010). En primer lugar, se pueden vender servicios de prevención para reducir o evitar los impactos ambientales a través de soluciones basadas en el conocimiento, la experiencia y el know-how, como los servicios de eficiencia energética. En segundo lugar, es posible vender servicios de mantenimiento y utilización de bienes a partir de la aplicación del principio de la responsabilidad ampliada del productor, tanto en negocios verticalmente integrados (en los que el productor asume la tutela y gestión del producto hasta el final de su uso) como en negocios que gestionan flotas (por ejemplo, de vehículos o maquinaria especial) o productos que pueden ser retornados a través de un sistema de logística inversa, como los envases. Por último, la venta de funcionalidad puede aplicarse a través de la remanufactura y ampliación de la vida útil de los bienes duraderos.

Como parte integral de la economía de servicios funcional, Stahel (2010) argumenta que los modelos de negocio deben centrarse en gestionar el desempeño o rendimiento a lo largo del tiempo para obtener beneficios de la duración de los bienes en lugar de obtenerlos por su venta. Por tanto, la realización de la EC y de la economía de servicios funcional reclama de las empresas, los consumidores y otros agentes una nueva forma de producir y consumir. A continuación, ponemos el foco en la servitización como estrategia innovadora basada en los servicios para contribuir a una economía más sostenible. 


\section{Servitización: de una estrategia para la competitividad al logro de mejoras medioambientales}

Tal y como hemos referido en un apartado anterior, los servicios pueden contribuir a una economía más sostenible por medio de su propia innovación o modificando actividades de distinta naturaleza con las que interaccionan. Un área de investigación reciente en la innovación de servicios es la que considera la fusión de productos y servicios. Sistemas producto-servicio (SPS), servitización y servicizing son diferentes términos para referirse a la estrategia basada en la hibridación de productos y servicios en una propuesta de valor para satisfacer las necesidades de los clientes. A lo largo de las dos últimas décadas se han ofrecido definiciones diversas $y$, en consonancia, se han investigado diferentes aspectos en torno a esta estrategia. La tabla 1 recoge los distintos enfoques de la literatura sobre la servitización y los SPS, junto con las principales referencias.

Tabla 1. Diferentes enfoques sobre la hibridación de productos y servicios

\begin{tabular}{l|l|l} 
& Enfoque & Referencias \\
\hline \multirow{2}{*}{ Servitización } & $\begin{array}{l}\text { Innovación y nuevo modo de funciona- } \\
\text { miento empresarial, orientado a mejorar la } \\
\text { competitividad empresarial }\end{array}$ & $\begin{array}{l}\text { Huikkola et al. (2016); Kohtamäki et al. } \\
\text { (2018); Neely, (2008); Rabetino et al. } \\
\text { (2016); Toffel (2002); Vandermerwe y Rada } \\
\text { (1988); Visnjic et al. (2016); Visnjic Kastalli y } \\
\text { Van Looy (2013) }\end{array}$ \\
\cline { 2 - 3 } & $\begin{array}{l}\text { Estrategia de innovación con impacto en la la } \\
\text { sostenibilidad corporativa }\end{array}$ & $\begin{array}{l}\text { Doni et al. (2019); Farías y Cancino (2021); } \\
\text { Seclen-Luna et al. (2021, 2020) }\end{array}$ \\
\hline \multirow{2}{*}{ Servicizing y SPS } & $\begin{array}{l}\text { Modelo de negocio sostenible con } \\
\text { impacto en la sostenibilidad desde una } \\
\text { perspectiva sistémica }\end{array}$ & $\begin{array}{l}\text { Goedkoop et al. (1999); Mont (2002); } \\
\text { Tukker (2004); Tukker y Tischner (2006); } \\
\text { White et al. (1999) }\end{array}$ \\
\cline { 2 - 3 } & Modelo de negocio para la EC & $\begin{array}{l}\text { Hidalgo-Carvajal et al. (2021); Michelini } \\
\text { et al. (2017); Pereira y Vence (2020); Yang } \\
\text { et al. (2018) }\end{array}$
\end{tabular}

Fuente: elaboración propia.

La servitización fue primero definida como un movimiento de las empresas "desde el antiguo y anticuado enfoque en los bienes o servicios hacia los "paquetes" o sistemas integrados [...], con los servicios en el papel principal» (Vandermerwe \& Rada, 1988, p. 314). Otras definiciones han apuntado el carácter innovador de la hibridación de productos y servicios, indicando que se trata de "un nuevo paradigma de funcionamiento empresarial» (Toffel, 2002), o de "la innovación de las capacidades y procesos de una organización» (Neely, 2008). Desde este enfoque, se considera a la servitización como una innovación orientada a la consecución de ventajas comerciales. Al respecto, Bryson (2010) señala:

La creciente importancia de la encapsulación de servicios pone de manifiesto la transformación en curso del proceso de producción capitalista, que trata de desarrollar nuevas formas de aumentar la rentabilidad. En este nuevo mundo, el consumo no es una transacción única entre el consumidor y el productor, sino que es el comienzo de una relación a largo plazo. Es a través de este tipo de relaciones a largo plazo que se desarrolla 
la confianza y el productor puede ser capaz de encerrar a los consumidores en su tecnología y sus servicios (p. 686).

El estudio de la servitización como medio para mejorar la competitividad de las empresas sigue siendo una importante línea de investigación (Baines et al., 2007; Huikkola et al., 2016; Kohtamäki et al., 2018; Neely, 2008; Rabetino et al., 2016; Visnjic et al., 2016; Visnjic Kastalli \& Van Looy, 2013). Algunas de las ventajas identificadas en la literatura son la posibilidad de obtener mayores rendimientos de una base instalada de productos con un largo ciclo de vida, mayores márgenes obtenidos de los servicios y mayor estabilidad de los ingresos, no sujetos a los ciclos de inversión y compra de materiales (Oliva \& Kallenberg, 2003). Estos beneficios superiores también dependen del potencial de innovación derivado del control de los productos y servicios durante su uso, la reducción de costes y recursos, la maximización de los resultados, la posibilidad de vender el conocimiento adquirido durante el proceso de desarrollo bajo la forma de servicios de consultoría y formación, y la reutilización de los productos en combinación con diferentes servicios (Beuren et al., 2013). Por supuesto, el aprovechamiento de las ventajas depende de la propia estrategia adoptada por la empresa y del modelo de negocio concreto (Furrer, 2010).

En una serie de estudios más recientes, la estrategia de servitización se ha analizado desde una perspectiva innovadora no solo en relación con sus efectos en la productividad, sino también respecto de la sostenibilidad corporativa. Basándose en una muestra de empresas manufactureras europeas, Doni et al. (2019) identifican una mejora en el consumo energético como resultado de la servitización, pero no en otros aspectos de la sostenibilidad corporativa. En diversos estudios de industrias manufactureras peruanas se demuestra el efecto de la estrategia de innovación orientada a producto-servicio en el logro de un mejor desempeño medioambiental (Seclen-Luna et al., 2020; 2021). Igualmente, un estudio de caso centrado en la industria hotelera chilena destaca el apoyo de la digitalización como soporte a la innovación basada en producto-servicio y la contribución a la sostenibilidad empresarial de los proyectos desarrollados (Farías \& Cancino, 2021).

Posteriormente, en línea con el planteamiento de Stahel (2010) acerca de la economía de servicios funcional, existe una corriente de la literatura que se centra en destacar las ventajas ambientales asociadas a los SPS. En el ámbito europeo, uno de los primeros documentos que menciona los SPS es un informe realizado para el Gobierno neerlandés que incluía el estudio de esta estrategia como parte de su política medioambiental (Goedkoop et al., 1999). Asimismo, casi en paralelo apareció un estudio de la Agencia Estadounidense para la Protección del Medio Ambiente sobre la servitización (White et al., 1999). En esta primera literatura se identifica el servicizing como una estrategia para desligar el crecimiento económico del consumo de recursos y energía, y de la degradación medioambiental. La lógica de este planteamiento reside en que el crecimiento económico ligado a los servicios, en la medida en que depende menos de las materias primas que circulan en la economía y más del valor creado percibido, puede ser una estrategia para la desmaterialización (Goedkoop et al., 1999). Para Mont (2002), "un SPS debería definirse como un sistema de productos, servicios, redes de soporte e infraestructura que se diseña para: ser competitivo, satisfacer las necesidades de los clientes y tener un impacto medioambiental inferior a los modelos de negocio tradicionales» (p. 239). Cabe indicar que el 
valor medioambiental se recoge también de manera explícita en otros conceptos similares como el de servicios ecoeficientes, que «mejoran la eco-eficiencia de las actividades de las empresas cliente» (Bartolomeo et al., 2003, p. 830); o el de los servicios domésticos sostenibles (Halme et al., 2004).

Los SPS pueden clasificarse a lo largo de un contínuum de productos a servicios: 1) Ios SPS basados en el producto, que consisten en comercializar productos con servicios adicionales como una forma de agregar valor. En estos modelos, las garantías de funcionamiento, los servicios de mantenimiento y los servicios de gestión del final de la vida útil están incluidos. 2) Los SPS orientados al uso, que son aquellos que facilitan a los clientes el acceso al producto y a su función, pero sin transferir la propiedad. Ejemplos de ello son los modelos centrados en alquiler y leasing de bienes, o los que facilitan el uso compartido de bienes. Estos permiten que un producto se convierta en un activo generador de unidades de funcionalidad, mientras que el acceso a los bienes puede darse de forma simultánea o sucesiva. 3) Los SPS orientados a resultados, que son aquellos que añaden valor mediante el suministro de una función, de tal forma que el cliente paga por el servicio recibido. Algunos ejemplos son la gestión de residuos (función tangible), la comunicación (función intangible) o la provisión de un ambiente (iluminación y climatización) (Tukker, 2004).

\subsection{Beneficios de la servitización para el medio ambiente y la EC}

En algunos SPS, los objetivos económicos y medioambientales pueden retroalimentarse (White et al., 1999) cuando el proveedor que internaliza los costes de uso y de la eliminación de los productos que fabrica tiene interés en aumentar la eficiencia de su producto reduciendo el uso de recursos, fabricando o utilizando un producto más duradero que requiera menos mantenimiento, o diseñando un producto reciclable o reutilizable. Cuando el producto tiene un valor económico al final de su ciclo de vida (componentes reutilizables o materiales valiosos, energía, mano de obra y capital), el productor puede encontrar incentivos para recuperar este valor a través de actividades de reciclaje, reacondicionamiento o reutilización. Por último, cuando el producto se convierte en una fuente de costos más que de beneficios, el productor o proveedor intentará reducir el número de unidades necesarias por unidad de servicio.

Con todo, a pesar de los supuestos beneficios medioambientales que pueden lograrse a través de los diferentes tipos de SPS (Tukker, 2004; Tukker \& Tischner, 2006), la evidencia empírica no es concluyente (Pereira \& Vence, 2020) y tampoco queda clara la contribución a la EC. La creación de valor circular de diferentes tipos de SPS puede lograrse a través de diversas estrategias (Yang et al., 2018), como la reutilización interna y el bucle de recuperación, o el bucle más largo y el uso en cascada, aprovechando coproductos y subproductos en otras cadenas de valor. Aunque cualquier tipo de SPS parece aumentar la tasa de reparación, reutilización, renovación y reciclaje, los modelos más prometedores son los que se centran en el uso y, sobre todo, en los resultados. Un análisis bibliométrico previo realizado por Michelini et al. (2017) también indicó que, en general, solo los SPS orientados a los resultados son eficaces en la realización de ecoeficiencia para la EC. 
La tabla 2 recoge la evidencia empírica sobre la contribución de los SPS a la sostenibilidad y la circularidad. Cabe señalar que los casos analizados se basan en marcos conceptuales diferentes, así como en metodologías diversas para la evaluación de las mejoras ambientales y de la circularidad. En algunos casos, orientados al uso o al resultado, se destaca la importancia del diseño para facilitar la reparabilidad, el reacondicionamiento y la refabricación (Bressanelli et al., 2018; Lindahl et al., 2014; Ræbild \& Bang, 2017). Los SPS orientados al uso se centran principalmente en activos de alto coste o en tecnologías de la información que requieren conocimientos y habilidades específicas para facilitar el servicio (Lelah et al., 2011; Lindström et al., 2017); por ello, son SPS que promueven la retención de la propiedad por parte de los fabricantes o de un intermediario de servicios. Algunos casos diferentes se dan en la industria de la moda, donde pequeñas empresas ofrecen servicios de alquiler de ropa (Ræbild \& Bang, 2017; Stål \& Jansson, 2017). La ampliación del valor de los recursos o el uso de subproductos y residuos a través de los SPS ha sido analizada en el sector agrícola (Devisscher \& Mont, 2008; Pereira et al., 2016), y también en casos centrados en la fabricación de productos cuya propiedad es retenida por los fabricantes y cuyo valor al final de su vida útil es alto o fácilmente recuperable (Bressanelli et al., 2018; Lindahl et al., 2014; Yang et al., 2018).

La servitización y su eficacia para la EC pueden sustentarse en la digitalización (Han et al., 2020). La eficacia del modelo "producto como servicio» en el sector automotriz radica en aspectos de coste, conveniencia y circularidad, junto a la relevancia de seis factores: el papel de la regulación para promover la EC en las empresas, la selección adecuada de materiales para evitar el residuo, el diseño para maximizar el valor del producto (a través de los servicios de reparación e, incluso, de reciclaje), la tecnología para la eficiencia energética, tener en cuenta la totalidad del ecosistema y aumentar la colaboración entre agentes para la coordinación de actividades (por ejemplo, el intercambio de conocimiento con el cliente emerge como una característica relevante).

Tabla 2. Contribución de los SPS a la sostenibilidad y la circularidad

\begin{tabular}{l|l|l} 
Referencia & Descripción del SPS & Principales hallazgos \\
\hline Bressanelli et al. (2018) & $\begin{array}{l}\text { Suscripción a esquemas de uso de } \\
\text { aparatos electrodomésticos (leasing) } \\
\text { con servicios de mantenimiento } \\
\text { predictivo y reparación }\end{array}$ & $\begin{array}{l}\text { La tecnología como facilitador de los } \\
\text { SPS orientados a uso: extensión de } \\
\text { la vida del producto y de los recursos } \\
\text { mediante el acceso a los productos y la } \\
\text { recuperación al final del uso }\end{array}$ \\
\hline Devisscher y Mont (2008) & $\begin{array}{l}\text { Cooperativa de servicios para los } \\
\text { productores de café: instalaciones } \\
\text { compartidas para transporte, uso en } \\
\text { común de maquinaria y servicios de } \\
\text { reparación }\end{array}$ & $\begin{array}{l}\text { Extensión del valor del producto me- } \\
\text { diante uso más intensivo y eficiente. } \\
\text { Mejoras económicas, sociales y } \\
\text { ambientales para la comunidad }\end{array}$ \\
\hline Lelah et al. (2011) & $\begin{array}{l}\text { Aplicación máquina-máquina para } \\
\text { registro de información sobre recogida } \\
\text { de residuos de vidrio }\end{array}$ & $\begin{array}{l}\text { Importancia de la mutualización de } \\
\text { infraestructuras, procedimientos de } \\
\text { instalación y mantenimiento para lograr } \\
\text { impactos ambientales }\end{array}$ \\
\hline
\end{tabular}




\begin{tabular}{|c|c|c|}
\hline \multirow[t]{3}{*}{ Lindahl et al. (2014) } & $\begin{array}{l}\text { Enchufes centrales para las papeleras: } \\
\text { servicios de acceso, mantenimiento y } \\
\text { recuperación }\end{array}$ & \multirow{3}{*}{$\begin{array}{l}\text { Mejoras ambientales logradas en com- } \\
\text { paración con los productos con base en } \\
\text { actividades de reciclaje, remanufactura, } \\
\text { reutilización y mantenimiento, así como } \\
\text { con planificación integral }\end{array}$} \\
\hline & $\begin{array}{l}\text { Limpieza de los exteriores de los edifi- } \\
\text { cios: método de lavado sin detergen- } \\
\text { te, planificación y operación integral }\end{array}$ & \\
\hline & Alquiler de compactadores de suelo & \\
\hline Lindström et al. (2017) & $\begin{array}{l}\text { Servicio técnico de información que } \\
\text { aumenta la eficiencia de la gestión del } \\
\text { reciclaje, optimizando el vaciado de los } \\
\text { contenedores a medida que se llenan } \\
\text { hasta el tope de su capacidad }\end{array}$ & $\begin{array}{l}\text { Servicio orientado a facilitar la EC, } \\
\text { aunque en sí mismo no sea circular. } \\
\text { Ayuda a evitar el desperdicio cuando se } \\
\text { sobrecargan los contenedores y a que } \\
\text { los camiones eviten recorrer kilómetros } \\
\text { innecesarios cuando estos aún no } \\
\text { necesitan ser vaciados }\end{array}$ \\
\hline Matschewsky (2019) & $\begin{array}{l}\text { SPS orientado al uso basado en servi- } \\
\text { cios de mantenimiento, recuperación } \\
\text { y uso compartido de maquinaria de } \\
\text { uso industrial }\end{array}$ & $\begin{array}{l}\text { Ciertas mejoras de eficiencia operativa, } \\
\text { extensión de la vida del producto y uso } \\
\text { más intensivo. Solo los SPS orientados } \\
\text { a resultados podrían lograr objetivos de } \\
\text { desacoplamiento absoluto en el uso } \\
\text { de recursos }\end{array}$ \\
\hline Pereira et al. (2016) & $\begin{array}{l}\text { Cooperativa de servicios agroganade- } \\
\text { ros: uso compartido de maquinaria, } \\
\text { servicios compartidos para la pro- } \\
\text { ducción y distribución de alimentos, } \\
\text { instalaciones y servicios compartidos } \\
\text { para la recría de ganado }\end{array}$ & $\begin{array}{l}\text { Extensión de la vida útil de la maqui- } \\
\text { naria y de las instalaciones comunes } \\
\text { mediante un uso más intensivo y pro- } \\
\text { fesional; servicios más ecoeficientes } \\
\text { para la obtención de alimentos y cría } \\
\text { de ganado }\end{array}$ \\
\hline Ræbild y Bang (2017) & $\begin{array}{l}\text { Servicio de suscripción para ropa de } \\
\text { bebé }\end{array}$ & $\begin{array}{l}\text { Reutilización circular de productos: di- } \\
\text { seño para la sostenibilidad y extensión } \\
\text { de la vida del producto mediante la } \\
\text { facilitación del acceso sin transferir la } \\
\text { propiedad }\end{array}$ \\
\hline \multirow[t]{3}{*}{ Stål y Jansson (2017) } & Arrendamiento de prendas de vestir & \multirow{3}{*}{$\begin{array}{l}\text { Extensión de la vida del producto } \\
\text { mediante el acceso sin transferir la } \\
\text { propiedad y extensión de la vida de los } \\
\text { recursos; sin embargo, los beneficios } \\
\text { de los SPS para la sostenibilidad no son } \\
\text { claros (efectos rebote) }\end{array}$} \\
\hline & $\begin{array}{l}\text { Asesoramiento para el lavado y kit de } \\
\text { reparación de prendas }\end{array}$ & \\
\hline & Sistema de intercambio de prendas & \\
\hline \multirow[t]{3}{*}{ Yang et al., 2018} & $\begin{array}{l}\text { Servicios técnicos añadidos a la venta } \\
\text { de unidades de productos para la in- } \\
\text { dustria pesada: mantenimiento regular } \\
\text { y reparación de los productos durante } \\
\text { la fase de uso }\end{array}$ & \multirow[t]{3}{*}{$\begin{array}{l}\text { Cada uno de los SPS crea distintas } \\
\text { forma de valor circular. Los SPS orien- } \\
\text { tados al uso y los resultados favorecen } \\
\text { la creación de valor circular; además, } \\
\text { retener la propiedad del producto } \\
\text { importa }\end{array}$} \\
\hline & $\begin{array}{l}\text { Arrendamiento de unidades de sepa- } \\
\text { ración de aire: reutilización, recupera- } \\
\text { ción, reacondicionamiento y reciclaje } \\
\text { de generadores de gas }\end{array}$ & \\
\hline & $\begin{array}{l}\text { Venta de gases industriales: reutili- } \\
\text { zación, recuperación, reacondiciona- } \\
\text { miento y reciclaje de generadores de } \\
\text { gas; y reutilización de coproductos y } \\
\text { subproductos (gases) }\end{array}$ & \\
\hline
\end{tabular}


No obstante, varios estudios cuestionan la contribución de los SPS a la EC. Esta no es una relación que pueda darse por garantizada ni de forma inintencionada, aunque pueden lograrse ciertas mejoras con respecto a los productos puros (Matschewsky, 2019; Doni et al., 2019). En una revisión de varios estudios empíricos, Pereira y Vence (2020) destacan la dificultad de concluir de forma general sobre la eficacia de los SPS para contribuir a una economía más circular debido a la escasa evidencia de casos, la diversidad de enfoques y métodos utilizados para medir los impactos, y los límites de los casos analizados. Por otra parte, algunos estudios evidencian la importancia de la colaboración para la transformación hacia la EC, reconociendo el alcance parcial y los límites de los resultados alcanzados por una única empresa (Parida et al., 2019).

Finalmente, cabe señalar que la servitización y los modelos de negocio para la EC se ven afectados por numerosos obstáculos, reflejo de la contradicción con el marco sistémico del modelo lineal industrial, la falta de tecnología y conocimiento, la ausencia de instituciones, etc. (Diaz et al., 2019; Tura et al., 2019). En un análisis sistemático de las principales barreras y facilitadores de la servitización en aras de la EC (Hidalgo-Carvajal et al., 2021), se hace hincapié en las estrategias enfocadas en el cliente y conducentes a la EC como las más apropiadas para la servitización. Igualmente, la servitización y la EC entrañan la existencia de conflictos entre la lógica propiamente empresarial de generación de beneficios económicos y los principios de la circularidad. Morales (2020) identifica varias tensiones paradójicas que afectan a los modelos de negocio circulares: el uso de residuos como recursos, el diseño de productos circulares, la mejora de la estética de los productos usados, la adecuación de la oferta y la demanda, el equilibrio de los costes en la EC y la gestión de las resistencias de la cadena de valor. Estas tensiones se añaden a las que ya habían indicado estudios previos para la servitización convencional, pues pasar de la pura venta de productos a modelos de servicios avanzados no siempre es una cuestión alcanzable o deseable para las empresas manufactureras (Gebauer et al., 2013; Helms, 2016; Kowalkowski et al., 2015; Neely, 2008; Paiola et al., 2013; Valtakoski, 2017; Visnjic et al., 2016).

\section{Conclusión: oportunidades para una EC basada en los servicios}

La EC establece un nuevo marco sistémico para la realización de las funciones de producción y consumo de forma más sostenible. Las actividades de servicios, dominantes en las economías contemporáneas, están llamadas a cumplir un papel relevante en la transformación de la economía y para posibilitar el asentamiento del nuevo paradigma de la EC. Para ello, es necesario que los propios servicios sean objeto de ecoinnovación, mejorando el desempeño ambiental de sus fuentes de materialidad (factores de producción empleados, espacios de producción y consumo, objeto o médium del servicio); pero, además, debe darse una vía de ecologización necesaria en los servicios para contribuir a la realización de los principios de la EC en su interacción con actividades de otra naturaleza (primarias e industriales). En este sentido, el conocimiento científico y técnico será un valor central de los nuevos servicios orientados a la EC. A continuación, realizamos una propuesta de servicios para la EC que parte de la consideración de las diferentes etapas de la vida de 
un producto, desde su diseño hasta su disposición final. Distinguimos, fundamentalmente, entre los servicios asociados a la producción, los servicios relacionados con la fase de uso y la recuperación final.

\subsection{Servicios asociados a la producción}

En la fase de diseño, serán fundamentales los servicios basados en conocimientos científicos y tecnológicos para concebir nuevos bienes y servicios, seleccionar materiales adecuados (renovables, reciclables, reciclados, no tóxicos) y crear procesos ecoeficientes, tanto en el aprovechamiento de materiales como de las fuentes de energía renovables. Esta fase es crucial, también, por su incidencia en las demás etapas, ya que en ella se podrán planificar los sucesivos ciclos de vida de los productos.

En la fase propiamente de producción, la incorporación de servicios para satisfacer las necesidades de los fabricantes debería ir en línea con las propuestas de la economía de servicios funcional. Los proveedores de bienes de equipo duraderos y de infraestructura pueden aplicar las diferentes opciones de SPS: servicios de reparación, actualización y remanufactura que permitan ampliar la vida útil y mejorar el desempeño de esos bienes; y servicios de utilización en común o compartida de ciertos bienes que permitan un mejor aprovechamiento de su capacidad. Igualmente, en línea con la tendencia a la externalización de ciertos procesos productivos, los servicios basados en el resultado podrían jugar aquí un papel primordial (realización de procesos específicos, logística inversa, etc.).

\subsection{Servicios relacionados con la fase de uso/consumo}

En esta fase, quizá las oportunidades de los servicios para cumplir con los principios de la EC y modificar los patrones de consumo serán incluso mayores. En primer lugar, debemos destacar la necesaria relevancia que deberían adquirir los servicios orientados a la extensión de la vida de los bienes de consumo: reparación, actualización y renovación. Estos servicios, ya tradicionales, deberían tomar un nuevo auge en una EC más sostenible, al igual que los mercados de reutilización y de productos de segunda mano.

También las formas de comercialización pueden variar a través de la prestación de servicios enfocados en facilitar el uso compartido (de forma simultánea o consecutiva) de ciertos bienes que permitan utilizar productos más sostenibles y eficientes, además de incrementar el aprovechamiento de su capacidad; por ejemplo, los vehículos para desplazamiento; las herramientas y aplicaciones de uso puntual, como las de jardinería; o los electrodomésticos de lavado y secado de ropa. El uso compartido también puede ser el foco de servicios especializados en la gestión de espacios generalmente infrautilizados que pueden tener un mayor aprovechamiento de su capacidad.

Los servicios orientados a resultados en la esfera del consumo pueden ser especialmente relevantes para la EC. Estos servicios deberían llevar a sus proveedores a buscar satisfacer las necesidades de los clientes de forma ecoeficiente y a dar mayor peso a la senda tecnológica basada en la información. Entre otros, podemos citar los centrados en ofrecer servicios de climatización e iluminación eficientes; y las plataformas de servicios de gestión documental, de contenidos, etc. 


\subsection{Servicios de recuperación al final de vida}

La EC busca cerrar los ciclos, de modo que el valor contenido en los productos y sus componentes al final de la vida útil pueda seguir aprovechándose. Por este motivo, los servicios centrados en la gestión de subproductos y residuos, la logística inversa, el desmontaje de productos, la clasificación de componentes de productos, etc., serán fundamentales. Como soporte, los servicios asociados a las tecnologías de rastreo y trazabilidad, la geolocalización, y el desarrollo de aplicaciones científicas y técnicas que permitan generar información, conocimiento y transparencia a lo largo de las cadenas de valor deberán tener también un importante desarrollo.

Sin ser exhaustiva, esta propuesta clasificatoria puede considerarse como un punto de partida para análisis más profundos de las actividades de servicio y su diseño para una EC más sostenible. Desde el punto de vista académico, cabe llevar a cabo una revisión y clasificación más comprensiva, así como su respectivo contraste con la evidencia empírica. Para las empresas, se debe tener en cuenta el marco y los principios de la EC, que se presenta como una oportunidad para diseñar sus actividades y estrategias de innovación, permitiéndoles mantener su competitividad en el largo plazo, de acuerdo con los límites biofísicos del planeta. 


\section{bibliografía}

Bartolomeo, M., Dal Maso,

D., De Jong, P., Eder, P.,

Groenewegen, P., Hopkinson, P.,

James, P., Nijhuis, L., Örninge,

M., Scholl, G., Slob, A., \&

Zaring, $\mathbf{O}$.

2003

Eco-efficient producer services-what are they, how do they benefit customers and the environment and how likely are they to develop and be extensively utilised? Journal of Cleaner Production, 11(8), 829-837. https://doi.org/10.1016/S09596526(02)00157-9

\section{Bessant, J., \& Bush, H.}

Innovation agents and technology transfer. En M. Boden e I. Miles (eds.), Services and the Knowledge-Based Economy (pp. 155-169) Londres: Continuum.

\section{Beuren, F. H., Gomes Ferreira, M. G., \& Cauchick Miguel, P. A.}

Product-service systems: a literature review on integrated products and services. Journal of Cleaner Production 47, 222-231. https://doi.org/10.1016/j jclepro.2012.12.028

\section{Bocken, N., Bakker, C., De}

Pauw, I., Bakker, C., \& Van der Grinten, B.

2016 Product Design and Business Model Strategies for a Circular Economy. Journal of Industrial and Production Engineering, 33(5), 308-320. https://doi.org/10.1080/216 81015.2016 .1172124

\section{Boden, M., \& Miles, I.}

Services and the Knowledge-based Economy (Science, Technology \& the Ipe). Londres: Continuum.
Bressanelli, G., Adrodegari, F., Perona, M., \& Saccani, N.

Exploring how usage-focused business models enable circular economy through digital technologies. Sustainability, 10(3). https://doi.org/10.3390/su10030639

\section{Bryson, J. R.}

2010 Service innovation and manufacturing innovation: bundling and blending services and products in hybrid production systems to produce hybrid products. En F. Gallouj y F. Djellal (eds.), The Handbook of Innovation and Services: A MultiDisciplinary Perspective (pp. 679-700). Cheltemham: Edward Elgar Publishing Limited.

\section{Cainelli, G., \& Mazzanti, M.}

2013 Environmental innovations in services:

Manufacturing-services integration and policy transmissions. Research Policy, 42(9). https://doi.org/10.1016/j. respol.2013.05.010

\section{Camacho, J. A., \& Rodríguez, M.}

Integración con la industria y actividad difusora en los servicios intensivos en conocimiento. En L. Rubalcaba Bermejo y H. Kox (eds.), Los servicios a empresas en el crecimiento económico europeo (pp. 179-196). Madrid: Marcial Pons.

\section{Cuadrado Roura, J. R., \&}

\section{Rubalcaba Bermejo, L.}

Los servicios a empresas en la industria española. Madrid: Instituto de Estudios Económicos.

\section{Devisscher, T., \& Mont, 0.}

2008 An Analysis of a Product Service System in Bolivia: Coffee in Yungas. International 


\section{bibliografía}

Journal of Innovation and Sustainable

Development, 3(3-4), 262-284

Diaz, F. J., Bastein, T., \& Tukker, A.

2019

Business model innovation for resource-

efficiency, circularity and cleaner

production: what 143 cases tell us.

Ecological Economics, 155, 20-35. https://

doi.org/10.1016/j.ecolecon.2018.03.009

\section{Djellal, F., \& Gallouj, F.}

2016

Service Innovation for Sustainability: Paths for Greening Through Service Innovation.

En Marja Toivonen (ed.), Service

Innovation. Translational Systems Sciences

(pp. 187-215). Tokio: Springer. https://doi.

org/10.1007/978-4-431-54922-2_9

\section{Doni, F., Corvino, A., \& Bianchi}

\section{Martini, S.}

Servitization and sustainability actions.

Evidence from European manufacturing

companies. Journal of Environental

Management, 234, 367-378. https://doi.

org/10.1016/j.jenvman.2019.01.004

\section{Ellen MacArthur Foundation (EMF).}

2013 Towards the circular economy: an

economic and business rationale

for an accelerated transition. https://

ellenmacarthurfoundation.org/towards-the-

circular-economy-vol-1-an-economic-and-

business-rationale-for-an

\section{Farías, A., \& Cancino, C. A.}

Digital Transformation in the Chilean

Lodging Sector: Opportunities for

Sustainable Businesses. Digital

Transformation in the Chilean Lodging

Sector. Opportunities for Sustainable

Businesses, 13(14), 1-17. https://doi.org/

https://doi.org/10.3390/su13148097
Furrer, $\mathbf{O}$.

2010

A customer relationship typology of product services strategies. En F. Gallouj y F. Djellal (eds.), The Handbook of Innovation and Services: A Multi-Disciplinary Perspective (pp. 701-721). Cheltemham: Edward Elgar Publishing Limited.

\section{Gadrey, J.}

The environmental crisis and the economics of services: the need for revolution. En F. Gallouj y F. Djellal (eds.), The Handbook of Innovation and Services: A Multi-Disciplinary Perspective (pp. 93-125). Cheltemham: Edward Elgar Publishing.

\section{Gallouj, F., \& Savona, M.} Innovation in services: a review of the debate and a research agenda. Journal Evolutionary Economics, 19, 149-172. https://doi.org/10.1007/s00191-008-0126-4

Gallouj, F., \& Weinstein, O. Innovation in Services. Research Policy, 26, 537-556

\section{Gebauer, H., Paiola, M., \& Saccani, N.}

Characterizing service networks for moving from products to solutions. Industrial Marketing Management, 42(1), 31-46. https://doi.org/10.1016/j. indmarman.2012.11.002

\section{Ghisellini, P., Cialani, C., \& Ulgiati, $\mathbf{S}$.}

A review on circular economy: the expected transition to a balanced interplay of environmental and economic systems. Journal of Cleaner Production, 114, 11-32. https://doi.org/10.1016/j.jclepro.2015.09.007 
Goedkoop, M. J., Van Halen, C. J. G., Te Riele, H. R. M., \& Rommens, P. J. M.

1999

Product Service systems, Ecological and Economic Basics. Report for the Dutch ministries of Environment (VROM) and

Economic Affairs (EZ). La Haya.

Halme, M., Jasch, C., \& Scharp, M.

2004 Sustainable homeservices? Toward household services that enhance ecological, social and economic sustainability. Ecological Economics, 51(1-2), 125-138. https://doi.org/10.1016/j. ecolecon.2004.04.007

Han, J., Heshmati, A., \&

Rashidghalam, $\mathbf{M}$.

2020 Circular Economy Business Models with a Focus on Servitization. Sustainability, 12(21), 1-17. https://doi.org/10.3390/ su12218799

\section{Helms, T.}

Asset transformation and the challenges to servitize a utility business model. Energy Policy, 91, 98-112. https://doi.org/10.1016/j. enpol.2015.12.046

\section{Hidalgo-Carvajal, D., Carrasco- Gallego, R., \& Morales-Alonso, G.}

From Goods to Services and From Linear to Circular: The Role of Servitization's Challenges and Drivers in the Shifting Process. Sustainability, 13(8). https://doi. org/10.3390/su13084539

Hipp, C., \& Grupp, H.
Research Policy, 34(4), 517-535. https://doi. org/10.1016/j.respol.2005.03.002

Huikkola, T., Kohtamäki, M., \& Rabetino, R.

2016 Resource realignment in servitization. Research-Technology Management, 59(4), 30-39. https://doi.org/10.1080/08956308.2 016.1185341

Kirchherr, J., Reike, D., \& Hekkert, M.

2017 Conceptualizing the circular economy: An analysis of 114 definitions. Resources, Conservation and Recycling, 127, 221-232. https://doi.org/10.1016/j. resconrec.2017.09.005

Kohtamäki, M., Baines, T., Rabetino, R., \& Bigdeli, A. Z.

2018 Practices and tools for servitization. Managing service transition. Palgrave Macmillan. https://doi.org/https://doi. org/10.1007/978-3-319-76517-4

Konietzko, J., Bocken, N., \& Hultink, E. J.

Circular ecosystem innovation: An initial set of principles. Journal of Cleaner Production, 253. https://doi.org/10.1016/j. jclepro.2019.119942

Korhonen, J., Honkasalo, A., \& Seppälä, J.

2018 Circular Economy: The Concept and its Limitations. Ecological Economics, 143, 37-46. https://doi.org/10.1016/j. ecolecon.2017.06.041

Kowalkowski, C., Windahl, C., Kindström, D., \& Gebauer, H. 


\section{bibliografía}

manufacturers' service-led growth

strategies. Industrial Marketing

Management, 45, 59-69. https://doi.

org/10.1016/j.indmarman.2015.02.016

\section{Kox, H., \& Rubalcaba, L.}

2009

La contribución de los servicios a empresas al crecimiento económico europeo. En Los servicios a empresas en el crecimiento económico europeo (pp. 115-138). Madrid: Marcial Pons.

Krausmann, F., Lauk, C., Haas, W., \& Wiedenhofer. D. From Resource Extraction to Outflows of Wastes and Emissions: The Socioeconomic Metabolism of the Global Economy, 1900-2015. Global Environmental Change, 52, 131-140. https://doi.org/10.1016/j. gloenvcha.2018.07.003

Larsen, J. N.

Supplier-user interaction in knowledgeintensive business services: types of expertise and modes of organization. En M Boden e I. Miles (eds.), Services and the Knowledge-Based Economy (pp. 146-154). Londres: Continuum.

Lelah, A., Mathieux, F., \&

Brissaud, D.

2011 Contributions to eco-design of machineto-machine product service systems: The example of waste glass collection. Journal of Cleaner Production, 19(9-10), 1033-1044. https://doi.org/10.1016/j.jclepro.2011.02.003

Lindahl, M., Sundin, E., \&

\section{Sakao, T.}

Journal of Cleaner Production, 64, 288-296.

https://doi.org/10.1016/j.jclepro.2013.07.047

Lindström, J., Hermanson, A., Hellis, M., \& Kyösti, P.

2017

Optimizing Recycling Management Using Industrial Internet Supporting Circular Economy: A Case Study of an Emerging IPS2. Procedia CIRP, 64, 55-60. https://doi. org/10.1016/j.procir.2017.03.072

\section{Matschewsky, J.}

2019

Unintended circularity?-Assessing a product-service system for its potential contribution to a circular economy. Sustainability, 11(10). https://doi. org/10.3390/su11102725

Michelini, G., Moraes, R. N., Cunha, R. N., Costa, J. M. H., \& Ometto, A. R.

From Linear to Circular Economy: PSS Conducting the Transition. Procedia CIRP, 64, 2-6. https://doi.org/10.1016/j. procir.2017.03.012

\section{Mont, 0.}

Clarifying the concept of product-service system. Journal of Cleaner Production, 10(3), 237-245. https://doi.org/10.1016/ S0959-6526(01)00039-7

\section{Morales, A. H.}

Exploring paradoxical tensions in circular business modelscases from North Europe. Sustainability, 12(18). https://doi. org/10.3390/su12187577

\section{Neely, A.}

Exploring the financial consequences of the servitization of manufacturing. Operations Management Research, 1 , 


\section{bibliografía}

103-118. https://doi.org/10.1007/s12063-

009-0015-5

\section{Oliva, R., \& Kallenberg, R.}

Managing the transition from products to services. International Journal of Service Industry Management, 14(2), 160-172. https://doi.org/10.1108/09564230310474138

Paiola, M., Saccani, N., Perona, M., \& Gebauer, H.

2013 Moving from products to solutions: Strategic approaches for developing capabilities. European Management Journal, 31(4), 390-409. https://doi. org/10.1016/j.emj.2012.10.002

\section{Parida, V., Burström, T., Visnjic,} I., \& Wincent, J.

Orchestrating industrial ecosystem in circular economy: A two-stage transformation model for large manufacturing companies. Journal of Business Research, 101, 715-725. https:// doi.org/10.1016/j.jbusres.2019.01.006

\section{Pereira, Á., Carballo-Penela, A.,} González-López, M., \& Vence, X.

A case study of servicizing in the farminglivestock sector: organisational change and potential environmental improvement. Journal of Cleaner Production, 124, 84-93. https://doi.org/http://dx.doi.org/10.1016/j. jclepro.2016.02.127

\section{Pereira, Á., \& Vence, X.}

Les Systèmes Produit-Service Comme

Business Models Pour I 'Économie

Circulaire: Potentialités et Limites.

Technologie et Innovation, 5(1), 1-22.

https://doi.org/https://www.openscience.

$\mathrm{fr} /$ Les-systemes-produit-service-comme-

business-models-pour--economie-circulaire
Rabetino, R., Kohtamäki, M., \& Gebauer, $\mathrm{H}$.

Strategy Map of Servitization. International Journal of Production Economics, 192, 144-156. http://dx.doi.org/10.1016/j. ijpe.2016.11.004

Ræbild, U., \& Bang, A. L.

2017 Rethinking the Fashion Collection as a Design Strategic Tool in a Circular Economy. The Design Journal, 20(sup. 1), S589-S599. https://doi.org/10.1080/146069 25.2017 .1353007

Reike, D., Vermeulen, W. J. V., \& Witjes, S.

2018 The circular economy: New or Refurbished as CE 3.0? - Exploring Controversies in the Conceptualization of the Circular Economy through a Focus on History and Resource Value Retention Options. Resources, Conservation and Recycling, 135, 246-264. https://doi.org/10.1016/j. resconrec.2017.08.027

\section{Seclen-Luna, J. P., Moya-} Fernández, P., \& Pereira, Á.

Exploring the effects of innovation strategies and size on manufacturing firms productivity and environmental impact. Sustainability, 13(6), 1-18. https://doi. org/10.3390/su13063289

\section{Seclen-Luna, J. P., Opazo-}

Basáez, M., Narvaiza, L., \& Moya Fernández, P. J.

Assessing the effects of human capital composition, innovation portfolio and size on manufacturing firm performance. Competitivness Review, 31(3), 625-644. https://doi.org/10.1108/CR-01-2020-0021 


\section{bibliografía}

\section{Stahel, W.}

2010

The Performance Economy (2da ed.).

Londres: Palgrave Macmillan.

\section{Stahel, W.}

2013

Policy for Material Efficiency-Sustainable

Taxation as a Departure from the Throwaway

Society. Philosophical Transactions. Series

A, Mathematical, Physical, and Engineering

Sciences, 371(1986), 1-19. https://doi.

org/10.1098/rsta.2011.0567

Stål, H. I., \& Jansson, J.

Sustainable Consumption and Value

Propositions: Exploring Product-Service

System Practices Among Swedish Fashion

Firms. Sustainable Development, 25(6),

546-558. https://doi.org/10.1002/sd.1677

\section{Toffel, M. W.}

2002

Contracting for Servicizing [Harvard

Business School Technology \& Operations

Mgt. Unit Research Paper Nº8-063].

https://doi.org/10.2139/ssrn.1090237

\section{Toivonen, $\mathbf{M}$.}

Different types of innovation processes in services. En F. Gallouj y F. Djellal (eds.), The Handbook of Innovation and Services: A Multi-Disciplinary Perspective (pp. 221-249). Cheltemham: Edward Elgar Publishing Limited.

\section{Toivonen, M., \& Tuominen, T.}

Emergence of innovations in

services. The Services Industry

Journal, 29, 887-902. https://doi.

org/10.1080/02642060902749492

\section{Tukker, A.}

Eight types of product-service system:

eight ways to sustainability? Experiences from SusProNet. Business Strategy and the Environment, 13(4), 246-260. https:// doi.org/10.1002/bse.414

\section{Tukker, A., \& Tischner, U.}

Product-services as a research field: past, present and future. Reflections from a decade of research. Journal of Cleaner Production, 14(17), 1552-1556. https://doi. org/10.1016/j.jclepro.2006.01.022

Tura, N., Hanski, J., Ahola, T., Ståhle, M., Piiparinen, S., \& Valkokari, P.

Unlocking circular business: A framework of barriers and drivers. Journal of Cleaner Production, 212, 90-98. https://doi. org/10.1016/j.jclepro.2018.11.202

\section{Valtakoski, A.}

2017

Explaining servitization failure and deservitization: A knowledge-based perspective. Industrial Marketing Management, 60, 138-150. https://doi. org/10.1016/j.indmarman.2016.04.009

Vandermerwe, S., \& Rada, J. Servitization of business: Adding value by adding services. European Management Journal, 6(4), 314-324. https://doi. org/10.1016/0263-2373(88)90033-3

\section{Vence, $\mathrm{X}$.}

Industria e innovación. Vigo: Xerais.

Vence, X., \& González-López, M.

Los servicios a empresas intensivos en conocimiento y los flujos externos de conocimiento en los sistemas de innovación abiertos. Ekonomiaz. Revista vasca de economía, 70, 184-205. https://www.ogasun.ejgv.euskadi.eus/ r51-k86aekon/es/k86aEkonomiazWar/ ekonomiaz/getArticulosPubl?idPubl=65 
bibliografía

Visnjic, I., Wiengarten, F., \&

Neely, A.

2016 Only the Brave: Product Innovation,

Service Business Model Innovation, and

Their Impact on Performance. Journal of

Product Innovation Management, 33(1),

36-52. https://doi.org/10.1111/jpim.12254

Visnjic Kastalli, I., \& Van Looy,

B.

2013 Servitization: Disentangling the impact

of service business model innovation

on manufacturing firm performance.

Journal of Opererations Management,

31(4), 169-180. https://doi.org/10.1016/j.

jom.2013.02.001

White, A. L., Stoughton, M., \& Feng, L.

1999 Servicizing: The Quiet Transition to

Extended Product Responsibility. http://

infohouse.p2ric.org/ref/17/16433.pdf

Yang, M., Smart, P., Kumar, M., Jolly, M., \& Evans, S.

2018 Product-service systems business models for circular supply chains. Production

Plannig \& Control, 29, 498-508. https://doi.

org/10.1080/09537287.2018.1449247

Fecha de recepción: 7 de junio de 2021 Fecha de aprobación: 4 de agosto de 2021 Fecha de publicación: 19 de noviembre de 2021 